\title{
The properties of the Gamma-Ray Blazars in the CJ-F VLBI sample
}

\author{
S.Britzen*, R.C. Vermeulen*, G.B. Taylor ${ }^{\dagger}$, R.M. Campbell ${ }^{+}$, \\ I.W. Browne ${ }^{\diamond}$, P. Wilkinson ${ }^{\diamond}$, T.J. Pearson ${ }^{`}$ and A.C.S. Readhead ${ }^{\imath}$ \\ *NFRA, P.O. Box 2, NL-7990 AA Dwingeloo, The Netherlands ${ }^{1}$ \\ $\dagger^{\dagger}$ NRAO, P.O. Box O, Socorro, NM 87801, USA; ${ }^{+}$JIVE, P.O. Box 2, NL-7990 AA Dwingeloo, \\ The Netherlands; ${ }^{\circ}$ NRAL, Jodrell Bank, Macclesfield, Cheshire SK11 9 DL, England UK; 'CIT, \\ Department of Astronomy, 105-24, Pasadena, CA 91125, USA
}

\begin{abstract}
We present first results from the analysis of multi-epoch VLBI observations of the EGRET detected sources [9] in the CJ-F sample (Caltech Jodrell-Flat-spectrum, [10]). These objects form a subsample of 14 sources within the 293 AGN of the full CJ-F sample. $5 \mathrm{GHz}$ VLBI snapshot observations of the CJ-F sources are continuously being performed in order to create a valid database for thorough statistical tests of pc-scale jet motion in AGN. All gamma-bright CJ-F AGN have been observed at least twice with the VLBA, which enables us to investigate jet component motions and paths. In particular, we concentrate on the analysis of those properties supposed to be essential for gamma-ray production, i.e., superluminal motion and bending. A paper discussing the possible relation between morphological changes and gamma-ray flaring/production is in preparation.
\end{abstract}

\section{INTRODUCTION}

With 66 high-confidence blazar identifications of sources detected by EGRET [9], it has become increasingly clear that $\mathrm{GeV}$ gamma-ray sources are preferentially radiobright, compact-core, flat-spectrum sources, many of which have been classified as optically violent variables (OVV) or blazars. The active galaxies Mrk 421 and Mrk 501 , and - at a lower level of significance - a few other AGN (some are members of the CJ-F sample), have been detected by ground-based Cherenkov telescopes at the highest-energy end of the electromagnetic spectrum accessible with current technology (TeV-regime). Superluminal motion is common to all $\gamma$-ray sources studied in sufficient detail (e.g., $[1,2,4,5]$ ). Many sources also exhibit a parsec-scale jet that is bent or is misaligned with respect to the kiloparsec-scale jet (see, e.g., [2]). As pointed out, for instance in [7], these facts suggest that strong gamma-ray emission and blazar properties are physically related. It has also been suggested that relativistic beaming may explain the observation that although all EGRETidentified AGNs are radio loud with flat spectra, not all radio-loud flat-spectrum AGNs are detectable gamma-ray sources. In order to test these hypotheses, we

1) This work was supported by the European Commission, TMR Programme, Research Network Contract ERBFMRXCT97-0034 CERES.

CP558, High Energy Gamma-Ray Astronomy, edited by F. A. Aharonian and H. J. Völk (C) 2001 American Institute of Physics 1-56396-990-4/01/\$18.00 
started an analysis of the properties of the gamma-bright blazars in the CJ-F sample - 14 CJ-F sources according to the third EGRET catalog [9].

\section{OBSERVATIONS AND RESULTS}

CJ-F, is a complete flux-limited sample of 293 flat-spectrum radio sources drawn from the $6 \mathrm{~cm}$ and $20 \mathrm{~cm}$ Green Bank Surveys $[8,12]$ with selection criteria as follows: $S(6 \mathrm{~cm}) \geq 350 \mathrm{mJy}, \alpha_{20}^{6} \geq-0.5, \delta(1950) \geq 35^{\circ}$, and $\left|b^{\mathrm{II}}\right| \geq 10^{\circ}$. A series of global VLBI and VLBA observations ( $5 \mathrm{GHz}$ ) of the CJ-F sources have been performed since 1990 . We believe that for unambiguous determination of the jet component position and motion parameters, it is necessary to have at least three observing epochs, spread over roughly 4 years. These observations are now $96 \%$ complete; for a subsample of 34 sources, the last epoch will be obtained this fall. This then completes the observational part of the survey. The data reduction is $92 \%$ complete. For 241 objects, three epochs of observations have now been obtained and reduced. For 32 sources, either no redshift information is available or the source reveals no structure. For the remaining 209 objects, jet component motion can be studied. In Table 1 we list the sources of the gamma-bright CJ-F subsample by EGRET name [9], the radio-source identification [9], the redshift, and an indication whether the results for this source are already included in Fig. 1.

TABLE 1. $\gamma$-bright sources in the CJ-F sample

\begin{tabular}{lllll}
\hline EGRET name & identified radio source & Comment & redshift & reduced \\
\hline 3EG J0222+4253 & $0219+428$ & & 0.444 & $\mathrm{y}$ \\
3EG J0721+7120 & $0716+714$ & poss. Id. & 1.43 & $\mathrm{y}$ \\
3EG J0808+4844 & $0804+499 ?$ & & 2.172 & $\mathrm{n}$ \\
3EG J0845+7049 & $0836+710$ & poss. Id. & 2.180 & $\mathrm{y}$ \\
3EG J0917+4427 & $0917+449 ?$ & & 0.901 & $\mathrm{n}$ \\
3EG J0952+5501 & $0954+556$ & & 0.368 & $\mathrm{y}$ \\
3EG J0958+6533 & $0954+658$ & & 0.031 & $\mathrm{y}$ \\
3EG J1104+3809 & MRK 421 & & 1.814 & $\mathrm{y}$ \\
3EG J1635+3813 & $1633+382$ & & 0.033663 & $\mathrm{y}$ \\
& MRK 501 & & 1.375 & $\mathrm{n}$ \\
3EG J1738+5203 & $1739+522$ & & 0.069 & $\mathrm{y}$ \\
3EG J2202+4217 & BL Lacertae & poss. Id. & 1.032 & $\mathrm{y}$ \\
3EG J2352+3752 & $2346+385 ?$ & & 1.992 & $\mathrm{y}$ \\
3EG J2358+4604 & $2351+456$ & &
\end{tabular}

The apparent velocities of components show a wide range of behaviour: different components in the same source can show different velocities; components can 
accelerate, decelerate, merge, or split; and in some cases a stationary component can co-exist with moving components. In Fig. 1 (top) we show the $\beta_{\text {app }}$ - redshift relation for all the 209 objects. Jet component speeds have been averaged for each source and only the absolute values are shown (calculations use $H_{0}=65 \mathrm{~km} \mathrm{~s}^{-1}$ $\left.\mathrm{Mpc}^{-1}, q_{0}=0.5\right)$. The tendency of higher $\beta_{\text {app }}$ at larger redshift, already visible in the former analysis (when most sources had only two epochs, [3]), can be confirmed; it even seems to be more pronounced with the additional data epoch here. We also confirm the lower $\beta_{\text {app }}$ for BL Lac objects as compared to those for quasars or galaxies [3]. A preliminary analysis of the average velocities of the (small) gammabright subsample indicates similar values as obtained for the gamma-quiet objects. One of the important results of the analysis of the present survey is that curved jet structures seem to be a very common phenomenon in AGN. In Fig. 1 (bottom) we show the curvature (in degrees) observed on pc-scales by VLBI plotted against redshift. Most sources reveal jets curved by less than 50 degrees, but in some sources curvature up to 100 degrees has been observed. In some cases quasi-oscillatory trajectories or ridge lines have been observed. Curvature is important with regard to the emission mechanisms that play a role in AGN (see below). It definitely has to be taken into account when calculating velocities, and might significantly change the motion statistics (not yet taken into account in Fig. 1) for the fast, strongly curved objects.

A final discussion of the results has to wait until the analysis has been completed. However, some pecularities might turn out to be significant:

-significant jet curvature is visible in all the $\gamma$-bright sources - the curvature for these seems to be higher than for the rest of the CJ-F sample (see Fig. 1, bottom). -multiple jet components in some sources follow a single smoothly bent path (e.g., 0219+428 and 0954+658). Also for the gamma-bright blazar PKS 0420-014 [5], which is not in the CJ-F, a smoothly bent jet path has been observed. In this source, the jet-components also all follow the same path.

A binary black hole model for PKS 0420-014 [6] is capable of explaining the motion of the VLBI components as well as the observed optical flaring (see also [11] for MRK 501).

From the analysis of the paths and velocities, we conclude that the orientation of the jet with respect to our line of sight is the most important factor in understanding the properties of AGN, because it controls which part of the jet we observe and how the jet components appear to move. A careful analysis of the whole CJ-F could potentially point towards the property(ies) that discriminate gamma-loud from gamma-quiet AGN.

\section{REFERENCES}

1. Barthel, P.D., Highlights Astron. 10, 551 (1995).

2. Bower, G.C., Backer, D.C., Wright, M., et al., ApJ 484, 118 (1997).

3. Britzen, S., Vermeulen, R.C., Taylor, G., et al., 1999a. In L.O. Takalo \& A. Si lanpää (eds.) BL Lac Phenomenon, ASP Conf. Ser. 159, 431. 
4. Britzen, S., Witzel, A., Krichbaum, T.P., et al., $A \mathscr{G} A \mathbf{3 4 1}, 418$ (1999b).

5. Britzen, S., Witzel, A., Krichbaum, T.P., et al., $A \& A$ A60, 65 (2000a).

6. Britzen, S., Roland, J., Laskar, J., et al., $A \& A$, accepted (2000b).

7. Fichtel, C.E., Bertsch, D.L., Chiang, J., et al., ApJS 94, 551 (1994).

8. Gregory, P.C., \& Condon, J.J., ApJS 75, 1011 (1991).

9. Hartman, R.C., Bertsch, D.L., Bloom, S.D., et al., ApJS 123, 79 (1999).

10. Taylor, G.B., Vermeulen, R.C., Readhead, A.C.S., et al., ApJS 107, 37 (1996).

11. Villata, M., Raiteri, C., $A \& A$ 347, 30i (1999).

12. White, R.L., \& Becker, R.H., ApJS 79, 331 (1992).

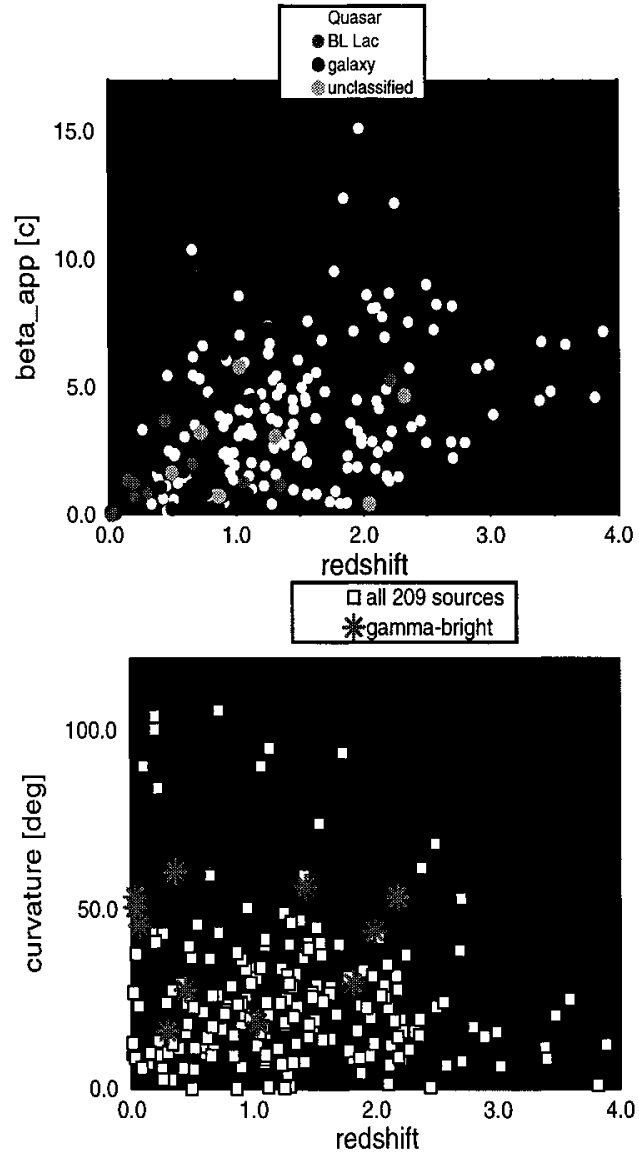

FIGURE 1. The figures shown above present the following relations: beta app (averaged over each jet) versus redshift for the 209 sources with at least three epochs of observations, and the curvature observed on VLBI scales versus redshift for both types of objects (white filled squares: gamma-quiet; red stars: gamma-bright CJ-F sources). The legends introduce the symbols used for different classes of objects. 